\title{
The Structure of a Quantum World
}

\author{
Fill North \\ (Forthcoming in The Wave Function, David Albert and Alyssa Ney, eds., \\ Oxford University Press) ${ }^{*}$
}

What is a world governed by quantum mechanics fundamentally like? In particular, what is the fundamental space of such a world like?

This question is puzzling. For the wave function-the thing that's governed by the dynamical laws, the object whose evolution predicts the results of quantum mechanical experiments-occupies a space that is very different from the one we seem to live in. The wave function's space has many dimensions-many more than the three dimensions of ordinary space (or four dimensions of ordinary space-time ${ }^{\mathrm{I}}$ ). Prima facie, realism about quantum mechanics seems to require realism about the wave function and the space it inhabits. But then there's a glaring question as to why, if our world is fundamentally quantum mechanical, we seem to live in a space of only three dimensions.

I argue for a view related to what has come to be called "wave function realism," though I wish to put the emphasis in a different place. Rather than starting from questions about the ontological status of the wave function (as do other authors who arrive at a view similar to the one I defend ${ }^{2}$ ), I want to focus on the fundamental space of a quantum mechanical world. Wave function realism will naturally go along with the view, but I will first argue for realism about the space on which the wave function lives. The reason is that there are some very general principles, familiar from elsewhere in physics, supporting the view that this space exists and is fundamental to such a world - that this is the fundamental physical space of such a world.

*I am grateful to David Albert, Otávio Bueno, Alyssa Ney, Ted Sider, Christian Wüthrich, audience members at the Pacific APA in 2010 and Calvin College in 201 2, and the Yale philosophy department for helpful discussion and comments on earlier versions of this essay.

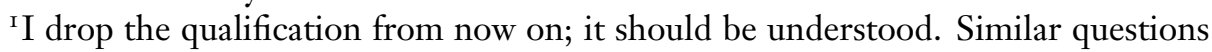
arise for four-dimensional space-time as for three-dimensional space.

${ }^{2}$ See especially Albert (1996). 
The question about the fundamental space of a quantum world is complicated in two ways that I leave aside here. First, the fundamental structure of a world's space(time) may be more properly given by a relativistic theory. Still, it is plausible that the fundamental theory of our world will be quantum mechanical. So it is worthwhile to think about what the world's fundamental space would be if ordinary quantum mechanics is its fundamental theory, leaving aside relativistic quantum mechanics or some other final theory. More important, similar considerations should carry over to the relativistic case; for ease of discussion, I limit this chapter to ordinary quantum mechanics of particles. 3

Second, there are different theories of quantum mechanics on the table. This will not affect the thrust of the discussion here, however. For all theories make central use of the wave function, and this suffices to generate the questions about space. Different theories disagree on whether there are other things in the world besides the wave function; and there is room for debate, on any theory, about what sort of thing the wave function is. Yet the discussion here takes off from the nature of the space that all these theories need to define the wave function. 4

${ }^{3}$ Wallace and Timpson (2010) argue that the case for configuration space realism weakens in quantum field theory because (among other reasons) particles are not fundamental and particle positions are imprecise, so the very idea of a configuration space is unclear. On my view, however, the fundamental space is the wave function's space, not configuration space; see section 2.

${ }^{4}$ All theories, realistically construed, regard the wave function as directly representing or governing some part of the fundamental ontology. Some theories, though, posit more in the fundamental ontology that what's represented in the wave function; Bohm's theory also posits particles (or one "world particle"; more later). And on some understandings of Bohm's theory - and some understandings of collapse theories like Ghirardi-Rimini-Weber (GRW) (Allori et al. (2008)) - the theory only posits particles in the physical ontology; the wave function then governs the particles. Different theories also disagree on the dynamics of the wave function. They all contain the Schrödinger equation as a fundamental law, but some theories also have an indeterministic law of wave function collapse, as in GRW. Bohm's theory contains an additional dynamical law, the guidance equation, though this can arguably be derived from the Schrödinger equation plus symmetry considerations. Many-worlds theories add nothing to the fundamental dynamics or ontology beyond the standard formalism and the wave function. On any theory, it is open to debate whether the wave function alone represents the fundamental ontology, or whether there are also, or instead, objects in ordinary space(time). There are different ways of construing the wave function- 
This chapter goes as follows. I first discuss the guiding principles I rely on, and the ways we use them in our scientific theorizing (section I). I then argue that these principles support the conclusion that the wave function's space is fundamental to a quantum world (sections 2 and 3 ). I end by suggesting that there is a way of reconciling the fundamentality of this high-dimensional space with the three-dimensionality of our experience (section 4). Note that for the purpose of this discussion, I assume realism about quantum mechanics, so that the wave function directly represents or governs (at least part of; see note 4) the ontology of a quantum mechanical world; I will not be arguing for this here.

I have a sneaking suspicion that there will ultimately be a stand-off between ordinary space and wave function space views, depending on what sort of evidence one chooses to weight most heavily: whether from ordinary experience or our usual inferences from fundamental physics (see the end of section 3). I suspect there is no conclusive argument that one type of evidence or the other must be primary-no conclusive argument for whether to privilege the manifest or scientific image of the world when these come into conflict. I present an argument intended to resolve the stand-off in favor of the scientific image, but I doubt this should convince the opponent who starts off prioritizing the manifest one. My goals here are more modest: to present a case for wave function space realism and to defray the most counterintuitive consequences of the view.

\section{The Dynamics as Guide to What Is Fundamental}

The principles I will use to argue for the fundamentality of the wave function's space can be summed up in slogan form like this: the dynamical laws are a guide to the fundamental nature of a world.

Spelling this out in more detail: how is a world built up, according to its fundamental physics?

At the fundamental level, there is the fundamental ontology of the theory, there is the space in which this ontology lives, and there is some

as a field, a law, a global property of particles, as belonging to another metaphysical category-but the wave function is invariably central. 
structure to that space. Then there are the dynamical laws, which say how the ontology evolves through this space over time.

This brings me to a very general principle that guides our physical theorizing, from which the other principles I use all extend: the dynamical laws are about what's fundamental to a world. The dynamical laws relate what's fundamental to what's fundamental, where what's fundamental includes the fundamental space and its structure, and the fundamental ontology. The dynamical laws govern the fundamental level of reality; that is why they are a guide to the fundamental nature of a world.

When I say that the laws "are a guide to the fundamental nature of a world," I mean that we infer the fundamental nature of a world from the dynamical laws. We do not directly observe the fundamental level of reality: we infer it from the dynamics. We posit, at the fundamental level, whatever the dynamical laws presuppose-whatever there must be in the world for these laws to be true of it. 5

Why emphasize the dynamical laws? Because physics is first and foremost about how and why physical objects move around and interact with one another, and the dynamical laws are generalizations describing this behavior. We thus infer the fundamental nature of reality from the dynamical laws, which are themselves inferred from the observable behaviors of physical objects. We posit, at the fundamental level, whatever is required for the laws governing objects' motions.

We are familiar with using this sort of reasoning - the reasoning that takes us from dynamical laws to the fundamental nature of the worldfor space-time. How do we infer the space-time structure of a world, according to its fundamental physics? We look at the dynamical laws and infer the structure that is needed to support the laws- "support" in the sense that the laws presuppose that structure; they cannot be formulated without assuming this structure. That is, we look at the mathematical space-time structure needed to formulate the theory and infer the corre-

${ }^{5}$ Compare Albert (I996) on the dimensionality of ordinary space. Albert suggests that we do not directly perceive that space has three dimensions. Rather, we see that there are three independent directions along which ordinary objects can approach one another and interact (and so there will be three different dimensions implicit in the dynamical laws governing these motions), and infer from this that ordinary space is three-dimensional. 
sponding physical geometry to the space-time of the world. In particular, we look at the dynamical laws formulated in a coordinate-independent, geometric way (coordinate-dependent formulations can sneak in extra structure that isn't really required) and consider the space-time structure needed to formulate the laws in this way. We then infer that this structure exists in a world governed by those laws. If the laws cannot be formulated without referring to some structure, then plausibly the structure must exist in a world governed by those laws.

In a classical mechanical world, for example, we infer that space-time is Galilean, not Aristotelian. Aristotelian space-time has all the structure of Galilean space-time, plus an additional preferred rest frame structure. Yet that further structure isn't needed or referred to by the dynamical laws: the laws are the same regardless of choice of inertial frame. This means that we can formulate the laws without assuming a preferred rest frame. So we infer that the space-time of the theory doesn't have this extra structure. If the laws were not invariant under changes in frame, on the other hand, then we would infer this structure, for the laws couldn't be formulated without it. (Compare: if the dynamical laws weren't invariant under space translations, we would infer that space has a preferred location. The laws could not be formulated without presupposing this.)

The rule to infer the space-time structure needed for the dynamical laws comes in two parts. First, we don't infer more space-time structure than what's needed for the dynamics-just as we don't infer Aristotelian space-time in a classical world. We infer the least space-time structure to the world that's needed to formulate the fundamental dynamics. Any additional structure is excess, superfluous structure, not in the world-as a choice of inertial frame in classical mechanics is an arbitrary choice in description, not a distinction in the world. Second, we infer at least as much structure as needed for the dynamics. We do not infer less than Galilean space-time structure in a classical world. The dynamical laws presuppose the distinction between straight and curved space-time trajectories, for instance, a distinction that is supported by Galilean space-time. ${ }^{6}$

\footnotetext{
${ }^{6}$ In North (2OI 2), I argue that classical space-time has a somewhat different fundamental structure than standardly supposed, but one that includes a fundamental affine (inertial, straight-line) structure.
} 
Thus, we infer the minimal structure required for the dynamics-we adhere to a minimize structure principle 7 -and we also infer at least as much structure as required - we adhere to a "don't eliminate too much structure" principle. In other words, we infer just that space-time structure required or presupposed by the fundamental dynamical laws. The idea behind this principle is intuitive: a match in structure between the dynamical laws and the world is evidence that we have inferred the correct space-time structure to a world governed by those laws. ${ }^{8}$

Notice that the same intuitive idea supports a principle to posit just that fundamental structure to the world-no more, no less—as needed to support the dynamical laws, regardless of whether this is the structure of an ordinary low-dimensional space(time) or some higher-dimensional space. Regardless, the match in structure between the dynamics and the world is evidence of our having inferred the correct structure to the world. Regardless, this principle stems from the very general idea that the dynamical laws are a guide to what is fundamental to a world.

Finally, we also infer the fundamental ontology presupposed by the dynamical laws. This may be less immediately familiar, but it is something we typically adhere to. Think of Newtonian physics. In addition to indicating the space-time structure, the dynamical laws tell us that, fundamentally, there are particles, which travel along straight paths unless acted on by a net external force. The dynamics presupposes that there are such things; the laws wouldn't be true if there weren't. So we infer, in a Newtonian world, that particles exist at the fundamental level. 9 Just as a match in structure between dynamics and world indicates that we have inferred the correct structure to the world, so a match in ontology between dynamics and world indicates that we have inferred the correct

\footnotetext{
${ }^{7} \mathrm{I}$ argue for this principle in North (2009).

${ }^{8}$ Compare Earman (1986, 26), (1989, 46).

${ }^{9}$ This isn't to say that Newtonian mechanics could not hold in a world of which matter is fundamentally gunky, or a world fundamentally containing only macroscopic objects. In the latter case, the objects can be treated as composed of point-sized bits or locations of matter to which the laws apply, even if there fundamentally are no particles; alternatively, the laws can be interpreted as governing the objects' centers of mass. In the former, it's unclear whether to consider the world genuinely Newtonian, though a version of Newton's laws can still hold. In typical Newtonian worlds, however, we make the inference to particles.
} 
fundamental ontology to the world.

In sum: we adhere to a general principle to infer just that fundamental structure and ontology that is required by the dynamical laws.

Three final notes on this principle. First, it is a guiding methodological principle; it will not yield conclusive inferences. We cannot be certain that the structure and ontology indicated by the dynamics is the correct structure and ontology of the world. We cannot be certain that there is no preferred frame in a classical world, for instance. Still, the principle is a reasonable guide, which we think has been successful. (Consider the inference to Minkowski space-time in special relativity. The special relativistic laws can be formulated without assuming absolute simultaneity, so it is reasonable to infer that there is no such structure in the worldreasonable, even though there could still be a preferred frame.)

Second, the form of the laws from which we read off the structure and ontology should be geometric. This is typically the simplest, most objective (coordinate-independent) statement of the laws, and thus the best guide to the nature of the world, apart from our descriptions of it.

Third, this principle applies to the fundamental level or supervenience base. It says to infer just that fundamental structure and ontology needed for the dynamics. ${ }^{10}$

In all, we infer the fundamental stuff the dynamical laws need in order to be geometrically formulated. In the next two sections, I suggest that we can use this general principle to figure out the fundamental space of a quantum mechanical world. And we can do so in a way that is less controversial than it might initially seem, given the principle's more familiar applications.

\footnotetext{
${ }^{10}$ We can think of this as an updated version of Quinean ontological commitment. Not: what there is, is what the values of the variables range over, so that we first render our theory in (first-order) logic and then see what the values of the variables are. Rather, what there fundamentally is, is given by the (best invariant formulation of the) dynamical laws, so that we first render our fundamental theory in geometric terms and then infer the structure and ontology presupposed by the laws.
} 


\section{The Fundamental Space of a Quantum World}

It's now a relatively short step to the conclusion that the wave function's space is fundamental to a quantum mechanical world.

In quantum mechanics, the wave function is the mathematical object that represents the state of a system at a time. ${ }^{\text {II }}$ Think of it as like a function and like a wave, as it is called. As with a function, the wave function takes in points of the space on which it's defined and gives out values, here complex numbers. As with a wave (or field), the wave function assigns a (complex) number, or amplitude (a "height"), ${ }^{\mathrm{I} 2}$ to each point in the space in which it lives. On the standard view, the wave function represents everything about the fundamental state of a system (where this system could be the entire world) at a time. (In Bohm's theory, the fundamental state is given by the wave function plus the positions of a system's particles.) A system's history is given by the evolution of its wave function over time, in accord with the dynamical laws. These laws include (at least) the deterministic Schrödinger equation. (Depending on the theory, there may be other fundamental dynamical laws; see note 4 . Ignore these complications here.)

The space on which the wave function is defined is high-dimensional: $3 n$ dimensions for a world containing what we ordinarily think of as $n$ particles in three-dimensional space. ${ }^{\mathrm{I} 3}$ (Whether there really are particles depends on the theory; more on this later.) This space, which I have been calling the wave function's space, is similar to what is called "configuration space," but these should not be confused. A configuration space represents ordinary particle configurations. Think of classical mechanics, where the configuration space of an $n$-particle system has $3 n$ dimensions, one for the location of each particle along each of three ordinary spatial dimensions; each point in this high-dimensional space represents a possible configuration of particles in three-space. A quantum mechanical configuration space is similar in that each point represents a

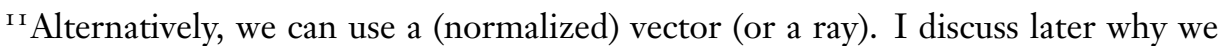
can set aside this other mathematical formulation here.

${ }^{\mathrm{I} 2}$ And phase, which I ignore here for convenience.

${ }^{13}$ I leave out spin for convenience. Spin can be represented by extra internal degrees of freedom at each point of the wave function's space.
} 
configuration of particles in ordinary three-dimensional space. In short, configuration space fundamentally represents particle configurations in three-space.

The view I defend is that the wave function's space is fundamental. Unlike configuration space, this high-dimensional space doesn't fundamentally represent particle configurations in three-dimensional space; the structure of this space isn't given by particle positions in three-space. The wave function's space, not three-space, is the fundamental space here. The wave function's space is isomorphic to configuration space, but it should not be confused with a genuine configuration space, in the sense of a space that fundamentally represents particle configurations in three-dimensional space. Fundamentally, there is no three-space on this view; a fortiori, fundamentally, there are no particles in three-space.

Now, in a theory like classical mechanics, configuration space is seen as just a mathematical tool. ${ }^{\mathrm{I}} \mathrm{H}$ The dynamics can be formulated either on the high-dimensional configuration space or a three-dimensional space. Ordinary experience then suggests that the three-dimensional space is the one that accurately represents the world's fundamental physical space.

In quantum mechanics, however, we must formulate the dynamics on a high-dimensional space. This is because quantum mechanical systems can be in entangled states, for which the wave function is nonseparable. Such a wave function cannot be broken down into individual threedimensional wave functions, corresponding to what we think of as particles in three-dimensional space. That would leave out information about correlations among different parts of the system, correlations that have experimentally observed effects. Only the entire wave function, defined over the entire high-dimensional space, contains all the information that factors into the future evolution of quantum mechanical systems. ${ }^{15}$

Following the principle to infer, at the fundamental level of the world,

\footnotetext{
${ }^{\mathrm{I}} 4$ But see North (2009, 20I2) against its being merely a mathematical tool.

${ }^{15}$ Thus (here speaking in terms of particles, though remember that on some views fundamentally there are no such things), two particles' locations might be perfectly correlated (always in the same region) or anticorrelated (in different regions). When projected onto the three-dimensional space for each particle, the wave function for such an entangled system looks the same whether the particles are correlated or anticorrelated. See Lewis (2004, section 2); Ney (2010a, section 3.3.), Ney (2010b); Lewis (2OI2) for this argument.
} 
just that structure and ontology that is presupposed by the dynamics, we are led to conclude that the fundamental space of a world governed by this dynamics is the high-dimensional one. The fundamental ontology, which includes the wave function, then lives in it. ${ }^{16}$ (Note that, on a wave function space version of Bohm's theory, the fundamental ontology also includes a "world particle," whose dynamical evolution in the wave function's space gives the evolution of the [non-fundamental; see section 4] "particles" in three-space.)

Of course, it is important to keep in mind the distinction between the wave function as a mathematical object and as a real physical fieldlikewise, between the abstract space on which the mathematical object is defined, and the physical space on which the physical field lives. So why not take the wave function and its space as mathematical tools that do not represent physical things in the world? Because of our guiding principle. This principle says to infer, from the mathematical structure needed to formulate the dynamical laws, the corresponding physical structure and ontology to the world. Compare the space-time case, in which we take a theory's mathematical structure seriously in that it corresponds to a particular physical geometry in the space(time) of the world.

(Another standard formulation of quantum mechanics uses a different mathematical space - an abstract vector space called Hilbert space. Unlike the wave function's space, though, this space is not a candidate fundamental physical space of a quantum world: Hilbert space is just a mathematical tool that yields a convenient formulation of the theory. Our general principle arguably yields this result. Recall that part of this principle warns against inferring too little fundamental structure to the world. One way to infer too little structure is by positing too minimal a basis on which to recover the ordinary world of our experience. The Hilbert space formulation seems to contain too little structure from which to construct a picture of the world as we experience it. Hilbert space does not support an objective, structural distinction between positions and other physical properties, like spin, in the way that the wave function's space does. ${ }^{7}$ In addition, the fundamental objects of

\footnotetext{
${ }^{16} \mathrm{Or}$, since on this view the wave function is a physical field, it may be better to say that it lives "on" this space; Maudlin (2010, I26).

${ }^{17}$ Compare Wallace and Timpson (2010, 703): "the physical universe is...very highly
} 
Hilbert space are vectors; the wave function space formulation, on the other hand, allows for a more familiar particle and field ontology (albeit a fundamentally very high-dimensional such ontology). Hilbert space is best interpreted as an abstract statespace; whereas the wave function's space can be interpreted as a physical space, inhabited by the fundamental physical objects of the theory. In all, it is too hard to recover a perspicuous picture of the world from the Hilbert space formalism. Of course, what counts as perspicuous is a matter of debate. Wallace and Timpson (2010) (and in a different way Maudlin (2007, 2010)) argue that wave function realism does not yield a perspicuous theory. I disagree, for reasons given in section 4.)

\section{Against Fundamental Three-Space Views}

Our general principle tells against views which maintain that a threedimensional space is fundamental to a quantum world. (Note that on any such view, the high-dimensional space of the wave function is a genuine configuration space.)

One view says there are two fundamental physical spaces, ordinary three-dimensional space and a high-dimensional configuration space. The wave function, in configuration space, governs the motions of particles (or other objects, such as mass densities or flashes (Allori et al., 2008); there will be some such fundamental objects, on this view) in three-space.

This theory has more structure than any view positing a single fundamental space. For there are two distinct fundamental spaces, each with its own structure. What's more, each space must possess additional structure beyond what is normally attributed to it. Further structure is needed to ground the connections between the two fundamental spaces, saying which parts and dimensions of the high-dimensional space correspond to which parts and dimensions of ordinary space, and which axes of configuration space correspond to which particle. Notice that this is additional fundamental structure. As such, it goes against our principle. This is extra fundamental structure beyond what is needed for the dynamics; it is excess structure we should do without. ${ }^{18}$

structured, whereas Hilbert-space vectors seem pretty much alike."

${ }^{\mathrm{I} 8}$ David Albert (in a seminar of Tim Maudlin's at Rutgers University in 2007) has 
David Albert noted ${ }^{\mathrm{I} 9}$ that the laws will also be odd on such a view. Ordinarily, we predict how things will behave by means of direct geometric relations among them. That is, all physical objects inhabit a single space, and the laws governing their motions and interactions are formulated in terms of the geometry of that space. Thus, consider the electromagnetic field and charged particles in classical electromagnetic theory. The direction and magnitude of the field everywhere determines, in accord with the dynamical laws, how the charges in various configurations will move around. We can look at the one space, figure out what direction the field is pointing in at a given location, and, using the laws, infer that a test particle will head in that direction when it passes through the location. This is unlike the two-fundamental-spaces view of quantum mechanics, in which the two kinds of thing in the world occupy distinct spaces, and so lack any direct geometric relations between them; the laws relate the wave function, in one space, to the particles, in another. (This is where the extra fundamental structure comes in, to say that this direction in configuration space corresponds to that direction in physical space, indicating how the wave function's behavior in its space causes the particles to move around in their space.) It's odd for fundamental laws to be formulated in terms of structure connecting distinct spaces, instead of the intrinsic geometry of a single space that everything inhabits. This is unlike other physical theories with which we are familiar, and it obfuscates our understanding of how the different objects interact.

The minimize structure principle says to infer that one space alone is fundamental. Since the dynamics requires the high-dimensional space of the wave function, we should infer that this space represents the fundamental physical space of a world governed by that dynamics. The defender of the two-fundamental-spaces view will reply that ridding the world of three-space is ridding the world of too much structure, against

raised a similar concern, arguing against what he calls the additional "metaphysical structure." Dorr (2009) is a version of the view. (See also the "mixed ontology" view of Monton (2002).) Dorr defends realism about a fundamental configuration space and a fundamental three-space, with fundamental "putting" relations connecting the two. It is hard to compare overall structure here: do further fundamental relations add fundamental structure? It seems to me that this will require more structure than any single-fundamental-space view, though I admit that this isn't clear-cut.

${ }^{19}$ At a conference at Rutgers University in 2007. 
the "don't eliminate too much structure" principle. Yet doing away with a fundamental three-space isn't yet to say that no such space exists: there could still be a nonfundamental three-space. I discuss this in section 4 .

(Why does the Hilbert space formulation abstract away too much, whereas a fundamental wave function space view does not? I have no conclusive reason for this. The thought is something like the following. It isn't abstracting away too much if you do not privilege the existence of ordinary objects at the fundamental level (see section 4); we learned that with the advent of atomic physics. But you should have room in your fundamental theory for drawing certain ordinary distinctions in some way or other; doing without such distinctions at the fundamental level would be abstracting away too much. Exactly how these distinctions are metaphysically accommodated does not matter. But it matters that you make some room for them in your fundamental theory. Otherwise, it will be too difficult to construct an ordinary picture of the world on the basis of the theory. Thus, the Hilbert space formulation abstracts away too much because it doesn't make room at the fundamental level for ordinary notions like position-it denies an objective distinction between, for example, spin facts and position facts-and it doesn't allow for a fundamental particle-field distinction. The wave function space formulation, on the other hand, does not abstract away too much by denying that ordinary objects exist at the fundamental level. More generally, there are certain kinds of facts without which a fundamental physical theory abstracts away too much, and certain other kinds of facts without which it doesn't. The best theory posits the structure and ontology required by the dynamics while allowing for enough ordinary facts and distinctions. This is admittedly vague, but I hope somewhat intuitive. ${ }^{2 \circ}$ )

Other views say that three-space alone is fundamental. Bradley Monton (2006, 20I2) argues that quantum mechanics is fundamentally about

\footnotetext{
${ }^{2}$ The same reasoning deems the Schrödinger picture, and not the Heisenberg one, a candidate for describing the fundamental nature of a quantum world, despite the mathematical equivalence. The Heisenberg picture lacks a metaphysically perspicuous picture of the world: there is just one physical state, unchanging in time; only the operators change. Maudlin (2010, I28-I29) discusses the ontological obscurity of this picture. Muller (1997a,b) discusses the equivalence between the Heisenberg and Schrödinger formalisms, and notes ways in which the resultant theories are inequivalent.
} 
particles in three-space. The wave function doesn't "live" on a physical space. It is a mathematical tool, defined on an abstract configuration space, which represents the quantum mechanical properties of ordinary particles.

This view faces a dilemma. Either Monton says that the quantum mechanical laws are about the wave function in configuration space, in which case he violates the principle that the dynamical laws are about what's fundamental, since for him the wave function and configuration space aren't fundamental. Or he says that the laws are about ordinary particles' properties, which are fundamental. But in that case the laws will likely be very complicated. (I say "likely" because he doesn't say exactly how the different quantum mechanical properties of particles are related to one another.) To be stated solely in terms of things that Monton takes to be fundamental, the laws must be formulated as constraints on ordinary particles' properties, like their locations in three-space. Yet it is hard to see how the quantum dynamics can be simply formulated in this way. The version of the laws we are familiar with employs the geometry of the high-dimensional space of the wave function. Monton's view also leaves us with a nagging question: what is it about particles' properties such that the abstract configuration space description is the right way to represent them? In all, this view lacks structure that's needed for the simple, geometric formulation of the dynamics.

Peter Lewis (2004) argues that quantum mechanical configuration space is fundamental, but that it has three dimensions in a relevant sense. This space is $3 n$-dimensional in requiring "that many independent coordinates to parameterize the properties of the system" $(2004,726)$. But in another sense, it is three-dimensional: the parameters needed to describe systems' fundamental states have a preferred grouping into threes. So there is a way of understanding everything that happens as unfolding in a space with three independent spatial directions. ${ }^{21}$

This does not alleviate the main problem for configuration space (or wave function space) realism, though. Even if configuration space is three-dimensional in some abstract, representational way-even if there

\footnotetext{
${ }^{21}$ Lewis (2OI2) goes on to argue that a quantum world is really three-dimensionalthat the sense in which configuration space is $3 n$-dimensional is misleading, and in any case non-spatial.
} 
is an abstract way of capturing what goes on in three dimensions-intuitively, the space of the theory is still really-fundamentally- $3 n$-dimensional, for this number of dimensions is needed to formulate the dynamical laws. ${ }^{22}$ The problem for wave function space realism stems from the fundamental geometry of this space, not the mathematical geometry of spaces we can use to represent it. The problem is that the fundamental geometry needed to formulate the theory, and the corresponding physical geometry we infer to the world, is not three-dimensional, contrary to what our experience suggests. That problem remains. (Ultimately, there may not be a deep disagreement here. I also think there is a sense in which a quantum world is three-dimensional: there exists a nonfundamental three-space [see section 4]. But I disagree that the wave function's space itself is three-dimensional in any sense; nor do I think that a quantum world is fundamentally three-dimensional. Whether there is a real disagreement depends on whether Lewis would agree with me that there is such a thing as the world's fundamental geometry, which can differ from the geometry of a nonfundamental space.)

Another view maintaining the fundamentality of three-space is Tim Maudlin's (2007). ${ }^{23}$ According to Maudlin, configuration space is a mathematical tool for defining the wave function, which governs the behavior of ordinary particles. ${ }^{24}$ The wave function in configuration spacealongside a world particle, in Bohm's theory-yields, in Maudlin's term, an informationally complete description, from which "every physical fact about the situation can be recovered" (2007, $3 \mathrm{I} 5 \mathrm{I})$. But this isn't an ontologically complete, "exact representation of all the physical entities and states that exist" (2007, 3 154). In other words, the wave function in con-

\footnotetext{
${ }^{22}$ In other words, the high-dimensional space is needed for a theory that's "dynamically complete," in the sense to be discussed shortly.

${ }^{23}$ Allori et al. (2008) (see also Allori (2OI 2)) is a relevantly similar view, though one that disagrees on the status of the wave function. Allori et al. (see also Sheldon Goldstein and Nino Zanghì (2OI 2)) suggest it is like a law (though its precise status depends on the particular theory of quantum mechanics); Maudlin (2007) refrains from putting the wave function in a particular category. In a talk I heard some years ago, Maudlin suggested that the wave function is unlike anything else, in its own metaphysical category.

${ }^{24}$ Or flashes or mass densities: Allori et al. (2008).
} 
figuration space is not an ontologically accurate (my phrase, not Maudlin's ${ }^{25}$ ) depiction of a quantum world, even though it allows us to predict everything that happens and so is informationally complete. The ontologically accurate description is instead given by particles in three-space (in Bohm's theory; alternatively, mass densities or flashes in three-space in $\mathrm{GRW}^{26}$ ), even though this isn't informationally complete (since that requires the wave function).

In general, Maudlin warns, we cannot assume that informationally complete descriptions are ontologically accurate. If we did, then we would eliminate charged particles from the ontology of classical electromagnetism, for instance, since there is an informationally complete description without them (because the divergence of the electric field suffices to give the charges' locations). Likewise, for any deterministic world, we would posit only whatever is in the world at one time, since the state at a time plus the laws is informationally complete. In the case of quantum mechanics, Maudlin argues, although the wave function in configuration space is needed for informational completeness, there are independent reasons - namely, the evidence from ordinary experiencefor positing three-dimensional objects, not the wave function, in the ontology. ${ }^{27}$

This brings us to a basic disagreement between wave function space and ordinary space views: how much to emphasize the dynamics in figuring out the fundamental nature of the world. Three-space views prioritize our evidence from ordinary experience, claiming that the world appears three-dimensional because it is fundamentally three-dimensional. Wave function space views prioritize our inferences from the dynamics, claiming that the world is fundamentally high-dimensional because the dynamical laws indicate that it is. Notice that, although this latter view is counterintuitive, there is precedent for the inference it relies on, as in

\footnotetext{
${ }^{25}$ I use the phrase because saying that the wave function isn't ontologically complete suggests that the wave function is in the ontology, it just isn't everything. In Maudlin's view, the wave function isn't in the physical ontology—it isn't a physical field.

${ }^{26}$ Though Maudlin (2010) gives considerations against the mass density picture.

${ }^{27}$ There can be informationally complete descriptions that are ontologically complete, like particles in classical mechanics. Maudlin suggests that whether informational and ontological completeness apply to the same description is to be decided on a case-by-case basis.
} 
the case of space-time structure discussed earlier. Indeed, we can rely on a similar inference for the ontology of classical electromagnetism, too. Although the field values will give the locations of the charges, as Albert has noted, ${ }^{28}$ this will not give their masses, which are also needed to predict particle and field value locations at other times. In other words, the field values aren't, in Albert's phrase, dynamically complete. That's why we do not eliminate charges from the ontology: they are required by the dynamical laws. (If the field description were dynamically complete, on the other hand, we might well conclude that charges aren't in the ontology. ${ }^{29}$ ) Wave function space views hew to this tradition of positing, in the fundamental level of the world, whatever is required by the dynamics.

Against Maudlin, then, I think that informational completeness of the right sort-dynamical completeness-does track ontological accuracy. A dynamically complete description contains the structure and ontology presupposed by the fundamental dynamical laws. $3^{\circ}$ For Maudlin, dynamical completeness and ontological accuracy can come apart: in quantum mechanics, there is dynamical information that doesn't correspond to any structure in the physical world. According to our general principle, however, the fundamental level of the world - the fundamental ontology, the fundamental space, and its structure-should contain whatever is required to formulate the dynamical laws; there should be a match between the structure needed for the dynamics and the fundamental structure of the physical world. If so, then we should infer that the fundamental physical space of a quantum world is the high-dimensional space of the wave function, with the fundamental ontology residing in it.

\section{The Structure of Appearances}

Assuming that our world is fundamentally quantum mechanical, there remains the question of whether wave function space realism can explain the fact that we appear to live in three-dimensional space.

\footnotetext{
${ }^{28}$ In a seminar at Columbia in 2008.

${ }^{29}$ There was a history of trying to do this, but it didn't work: Arntzenius (1993).

${ }^{3} \mathrm{On}$ this understanding, the state at one time in a deterministic world is not dynamically complete. For the laws relate states at different times, thereby presupposing that there are such states.
} 
Maudlin (2007) argues that it can't, because it lacks what J. S. Bell calls "local beables" (1987, 52-53), parts of the ontology localized to regions of ordinary space(time). Indeed, Maudlin says, it is hard to see how we could ever come to understand, let alone empirically confirm, such a theory, when all of our evidence takes the form of local beables. ${ }^{3}$

Now, it is true that there are no fundamental local beables, on this view. But this doesn't mean that there are no nonfundamental such things. Indeed, I think that something like this holds for three-space and its objects as a whole. Unlike Albert (I996), who argues that in a quantum world, ordinary space is an "illusion" and our talk about it is false, ${ }^{32}$ I think that three-space exists in such a world, and our talk about it is true. It's just that this space is nonfundamental. Similarly, ordinary particles exist but are nonfundamental. They are more like tables and chairs: made up out of fundamental stuff, not themselves in the fundamental inventory. 33

In place of Albert's antirealism about three-space, I suggest an antifundamentalism. Ordinary space exists at a "higher level." Even so, there are objective facts about it and we can say true things about it. It's just that none of this is fundamental-just as tables and chairs exist and have objective truths about them but are not fundamental. We might say that statements about these things aren't strictly speaking true, but this just means that they are not fundamentally true. There is an objective fact as to where something is located in three-space, even though there is no such fundamental fact.

There are tricky issues here about exactly how to understand the claim that ordinary space exists but is nonfundamental. On some recent views in metaphysics, we cannot make sense of such claims; we must say that the nonfundamental things simply do not exist.

I think that there is a way of making sense of the idea that ordinary space is nonfundamental yet real, in the same way that ordinary objects, the special sciences, and so on, are nonfundamental yet real. A grounding

${ }^{3 \mathrm{r}}$ See Maudlin (2010) for further argument along these lines.

${ }^{32} \mathrm{Ney}(2 \mathrm{OIOb})$ in a different way argues that three-dimensional space doesn't exist in a quantum world.

${ }^{33}$ Compare Albert and Loewer (I995) and Wallace (2003, 2010). 
relation 34 captures the way that the wave function's space is fundamental and ultimately responsible for ordinary space, while at the same time allowing for the reality of ordinary space. This is an explanatory relation that captures the way in which one thing depends on or holds in virtue of another, without implying that the dependent thing does not exist. Thus, three-dimensional space and its objects are grounded in the wave function's space and its objects. For example, there being a table in threespace consists in nothing but the wave function having a certain shape in its high-dimensional space. It's true that there is a table in three-space; it's just that this holds in virtue of some other, more fundamental facts. The truth about three-space (the grounded) is not a further fact beyond the truth about the wave function's space (the grounds) - that is, it isn't a fundamental fact—even though it is distinct from the grounds and is itself a real fact.

More generally, the wave function's space is fundamental, and threespace is grounded in it; what's true of three-space holds in virtue of what's true of the wave function's space. This captures the way that three-space is emergent but "no less real for that" (Wallace and Timpson, 2010, 706). It also captures the idea that three-dimensional happenings are nothing over and above various wave function space happenings; that is, that three-space is not fundamental. In the way that thermodynamic or biological happenings, say, are nothing over and above various particle happenings - the former processes are grounded in more fundamental particle processes-so, too, for ordinary three-space happenings vis-àvis what goes on in the wave function's space. (Thus, the grounding relation more generally captures the way in which there are ordinary macrolevel sciences, with generalizations that are objectively true. They just aren't fundamental, but hold in virtue of what goes on at the fundamental level.)

I submit that this is the overall simplest, empirically adequate account of a quantum world. It explains our experience and captures the truth of our ordinary claims about three-dimensional space, while at the same time positing just that structure that's needed for the dynamics. 35 This

\footnotetext{
${ }^{34}$ Say, like that of Fine (200I); what I say here is neutral on the metaphysics of grounding.

${ }^{35}$ Fine $(200 \mathrm{I}, 22)$ notes that we can evaluate a system of grounds "in much the same
} 
view has the benefit of a fundamental three-space view-what explains the fact that the world appears three-dimensional is that there exists a three-dimensional space-while also matching the structure and ontology for the dynamics. (What it doesn't do is explain the fact that threespace appears fundamental by saying that three-space is fundamental.) In particular, there is no fundamental structure beyond what is needed for the dynamics. For there is no fundamental structure connecting ordinary space and the wave function's space; there are simply "grounding rules" from the fundamental to the nonfundamental, and these do not add fundamental structure, in the same way that correspondence rules for the special sciences don't add fundamental structure to the world. This picture also avoids the worry raised by Monton (2006) that the view is radically revisionary. It is indeed fundamentally revisionary, but it is not revisionary about the nonfundamental.

You might wonder why the wave function's space grounds an emergent three-dimensional space, not some other. Monton (2006) argues that it doesn't manage to do this, because there is no intrinsic structure in the wave function's space marking out a preferred grouping of axes into threes. There is nothing special about the fact that the number of dimensions is equal to " 3 times $n$ ": this space isn't fundamentally about particles in three-space.

But we have more to work with than just the kinematical structure of the space. There is also the dynamical structure, and this opens avenues of response. Lewis (2004) (see also Lewis (2012)) argues that the wave function's space does have intrinsic structure picking out a preferred grouping of dimensions into threes. Albert (1996) alternatively suggests that the form of the Hamiltonian results in the illusion of three-space, without extra intrinsic structure. Albert argues that the Hamiltonian has a uniquely natural form in three dimensions; in my view, this naturalness is evidence that the grounded space is, in fact, three-dimensional. Wallace and Timpson (2010) agree with Albert's point, adding that the experience of three dimensions should emerge due to decoherence. $3^{6}$

I suspect that one of these views is correct. One of these can explain

way as any other explanatory scheme, on the basis of such considerations as simplicity, breadth, coherence, or non-circularity" and most importantly, "explanatory strength."

${ }^{36}$ Though they argue that this is insufficient to fully recover the world. 
how the fundamental facts about the wave function (and perhaps a world particle) in the high-dimensional space ground the three-dimensional facts. Even if not, though, we could take this as some additional fundamental structure. This structure isn't needed for the dynamics. But there is more guiding theory choice than just the "posit what's needed for the dynamics" rule. There is also empirical adequacy, which may require a primitive "preferred grouping of axes into threes" structure. That is, the wave function's space itself may have an additional level of structure marking where its dimensions group themselves into threes; this would then ground the three-space facts. Even so, this view is preferable overall. For it has just about the fundamental structure needed for the dynamics, while also explaining how the fundamental facts ground the three-dimensional facts. Notice that on any of these approaches, this way of grouping the dimensions of the fundamental space is the right way of doing so, because it captures the truths about the non-fundamentaljust as there is a correct way of carving up the fundamental statespace into macroscopic parameters, namely the way that yields the truths about the higher-level sciences. Of course, none of this is to say exactly how the grounding of three-space in the high-dimensional space occurs; that question remains. But it answers the objection from local beables.

Finally, you might worry that the structure of the wave function's space remains unexplained on this view. I cannot say that it has the structure and dimensionality it does because it represents particle configurations in three-dimensional space. It isn't fundamentally about particles in three-space.

According to my view, however, the structure of the wave function's space is fundamental, not in need of explanation on the basis of anything more fundamental. We infer this structure from other things, like the dynamical laws; but this space has the structure it does because the world fundamentally is the way that it is. It may seem remarkable that it has just the right structure to yield the appearance-and the existenceof a three-dimensional space. But of course it does, if this really is the fundamental theory and those are the appearances that the theory saves.

Think of it this way. The relation between the wave function's space and its ontology, on the one hand, and three-dimensional space and its ontology, on the other, is analogous to the relation between particles, 
on the one hand, and tables and chairs, on the other. Compare: isn't it remarkable, if particles are fundamental, that they should conspire to make it seem as though there really are tables and chairs? But of course particles conspire to form themselves into tables and chairs, if particles really are in the fundamental level of reality and the nonfundamental stuff includes tables and chairs. Since the apparent existence of tables and chairs is the starting point for our theorizing, of course the fundamental theory we are led to is one that predicts the appearances (and existence) of tables and chairs. To put it another way, our evidence for the theory, in the first place, is what we observe. But what we observe, everyone agrees, is a parochial reflection of our own situation: we are familiar with tables and chairs. It is then no great coincidence that we end up with a fundamental theory that has the power to predict the appearances for us.

\section{Conclusion}

Why conclude that wave function space realism (or wave function space fundamentalism) is a physically accurate picture of a quantum world? Why not think the wave function's space is just part of the mathematics used to formulate the theory? Because we generally posit, in the physical world, the fundamental structure and ontology presupposed by the dynamical laws. This match between dynamics and world is evidence that this is the fundamental nature of a world governed by that dynamics.

\section{References}

Albert, David and Barry Loewer ( I 995). "Tails of Schrödinger's Cat." In R. Clifton, ed., Perspectives on Quantum Reality, pp. I8 I-I92. Kluwer.

Albert, David Z. (1996). "Elementary Quantum Metaphysics." In J. T. Cushing, A. Fine, and S. Goldstein, eds., Bohmian Mechanics and Quantum Theory: An Appraisal, pp. 277-284. Dordrecht: Kluwer Academic Publishers.

Allori, Valia (2012). "Primitive Ontology and the Structure of Funda- 
mental Physical Theories." In Alyssa Ney and David Albert, eds., The Wavefunction. New York: Oxford University Press.

Allori, Valia, Sheldon Goldstein, Roderich Tumulka, and Nino Zanghì (2008). "On the Common Structure of Bohmian Mechanics and the Ghirardi-Rimini-Weber Theory." British Fournal for the Philosophy of Science 59(3), 353-389.

Arntzenius, Frank (1993). "The Classical Failure to Account for Electromagnetic Arrows of Time." In T. Horowitz and A. Janis, eds., Scientific Failure, pp. 29-48. Maryland: Rowman \& Littlefield Savage.

Bell, John S. (1987). Speakable and Unspeakable in Quantum Mechanics. Cambridge, UK: Cambridge University Press.

Dorr, Cian (2009). "Finding Ordinary Objects in the World of Quantum Mechanics." Unpublished manuscript.

Earman, John (1986). A Primer on Determinism, vol. 32. University of Western Ontario Series in the Philosophy of Science: Reidel.

—_ ( 1989). World Enough and Space-Time. Cambridge, MA: MIT Press.

Fine, Kit (200I). "The Question of Realism." Philosopher's Imprint I.

Goldstein, Sheldon and Nino Zanghì (2012). "Reality and the Role of the Wavefunction in Quantum Theory." In Alyssa Ney and David Albert, eds., The Wavefunction. New York: Oxford University Press.

Lewis, Peter (2004). "Life in Configuration Space." British fournal for the Philosophy of Science 55, 7 1 3-729.

_- (20I2). "Dimension and Illusion." In Alyssa Ney and David Albert, eds., The Wavefunction. New York: Oxford University Press.

Maudlin, Tim (2007). "Completeness, Supervenience, and Ontology." Fournal of Physics A: Mathematical and General 40, 3 I 5 I-3 I 7 I. 
_ (20I0). "Can the World Be Only Wavefunction?" In Simon Saunders, Jonathan Barrett, Adrian Kent, and David Wallace, eds., Many Worlds?: Everett, Quantum Theory, and Reality, pp. I2 I-I43. Oxford: Oxford University Press.

Monton, Bradley (2002). "Wave Function Ontology." Synthese I 30, 265277 .

_ (2006). "Quantum Mechanics and 3 N-Dimensional Space." Philosophy of Science 73(5), 778-789.

- (2OI 2). “3N-Dimensional Space and Experience.” In Alyssa Ney and David Albert, eds., The Wavefunction. New York: Oxford University Press.

Muller, F. A. (I 997a). "The Equivalence Myth of Quantum MechanicsPart I." Studies in History and Philosophy of Modern Physics 28(I), 35-6I.

(I997b). "The Equivalence Myth of Quantum Mechanics-Part II." Studies in History and Philosopby of Modern Physics 28(2), 2 I 9-247.

Ney, Alyssa (2010a). "Are There Fundamental Intrinsic Properties?" In Allan Hazlett, ed., New Waves in Metaphysics, pp. 2 I9-2 39. New York: Palgrave Macmillan.

- (20Iob). "The Status of our Ordinary Three Dimensions in a Quantum Universe." Nô̂s pp. doi: IO.I I II/j.I4680068.2010.00797.x.

North, Jill (2009). "The 'Structure' of Physics: A Case Study." Fournal of Philosophy 106(2), 57-88.

— (2OI2). "Structure in Classical Mechanics." Unpublished manuscript.

Wallace, David (2003). "Everett and Structure." Studies in History and Philosophy of Modern Physics 34, 87-IO5. 
- (2010). "Decoherence and Ontology." In Simon Saunders, Jonathan Barrett, Adrian Kent, and David Wallace, eds., Many Worlds?: Everett, Quantum Theory, and Reality, pp. 53-72. Oxford: Oxford University Press.

Wallace, David and Christopher G. Timpson (2010). "Quantum Mechanics on Spacetime I: Spacetime State Realism.” British fournal for the Philosophy of Science 6I, 697-727. 